\title{
Nanoparticle-Assisted Surface Immobilization of Phospholipid Liposomes
}

\author{
Liangfang Zhang, Liang Hong, Yan Yu, Sung Chul Bae, and Steve Granick* \\ Departments of Chemical and Biomolecular Engineering, Materials Science and Engineering, Chemistry, and \\ Physics, University of Illinois, Urbana, Illinois 61801
}

Received April 21, 2006; E-mail: sgranick@uiuc.edu

Liposomes are artificially constructed spherical lipid vesicles with a small size, whose controllable diameter from tens to thousands of nanometers signifies that individual liposomes comprise compartments with volumes from zeptoliters $\left(10^{-21} \mathrm{~L}\right)$ to femtoliters $\left(10^{-15} \mathrm{~L}\right)$ that can be used to encapsulate and store various cargoes, such as proteins, DNA, and drug molecules. ${ }^{1-3}$ When biomolecules are loaded inside, dynamical behavior such as protein foldingunfolding transitions, enzyme reaction kinetics, and pathways can be observed inside the liposome on the single-molecule level.,5 Compared to studying individual biomolecules in buffer solution or those immobilized directly to a solid surface, trapping single biomolecules in surface-immobilized liposomes has advantages: (i) a biocompatible environment, (ii) prolonged observation time for each molecule, whose residence time within a laser focus volume would otherwise usually be brief due to its fast diffusion in bulk solution, ${ }^{6}$ and (iii) lesser surface perturbation (electrostatic interaction, steric hindrance, etc. $)^{7}$ on single molecule dynamics as vs the case for molecules immobilized on a surface directly.

There is a severe limitation, however. When liposomes encounter planar solid substrates, they fuse to form a planar supported bilayer if the solid is hydrophilic or a planar monolayer if the surface is hydrophobic. ${ }^{89}$ Several chemically based methods have been proposed to overcome this. Boxer and co-workers used oligonucleotide pairs to tether liposomes to solid-supported planar bilayers. ${ }^{10,11}$ Biotin-streptavidin linkers to solid-supported bilayers have been employed. ${ }^{12}$ Höök et al. found that cholesterol-modified ss-DNA can also anchor liposomes to solid-supported bilayers. ${ }^{13}$ While these pioneering methods are effective, it is also desirable to develop an immobilization scheme that is simpler chemically and applies generally to immobilize liposomes onto bare solid surfaces that can equally well be hydrophilic or hydrophobic.

Here we show that stabilization with adsorbed nanoparticles meets this need. The simple strategy of mixing phospholipid liposomes with charged nanoparticles and using low-power sonication to mix them at low volume fraction produces particlestabilized liposomes that repel one another and do not fuse, while leaving uncovered a large $(\approx 75 \%)$ free membrane outer surface. ${ }^{14}$ The particle-stabilized liposomes carry a net electric charge but can be manufactured using lipids that carry no net charge. Previous investigation of nanoparticle-stabilized vesicles addressed their behavior in bulk suspension. ${ }^{14}$ Now we consider their surface immobilization for what is considered to be the first time.

For study, phospholipid DLPC, 1,2-dilauroyl-sn-glycero-3-phosphocholine, was selected because its gel-to-fluid phase transition of $-1{ }^{\circ} \mathrm{C}$ was far below the experimental temperature, $23{ }^{\circ} \mathrm{C}$. We prepared small unilamellar lipid vesicles (liposomes) with a diameter of $200 \mathrm{~nm}$ in PBS buffer (10 mM, pH = 6.0) using the well-known extrusion method, employing procedures described in detail elsewhere. ${ }^{15}$ The fluorescent lipid was DMPE, 1,2-dimyristoyl-snglycero-3-phosphoethanolamine, with the polar headgroup labeled by rhodamine B (DMPE-RhB). Both lipids were obtained from

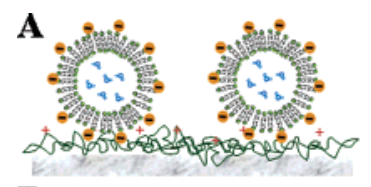

B
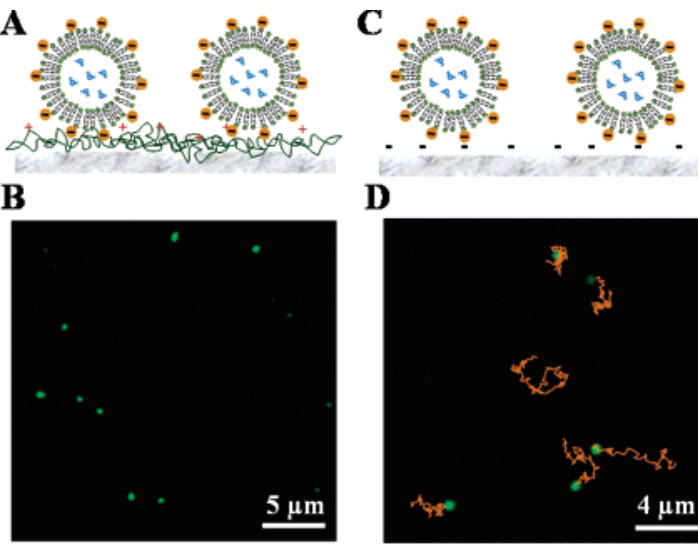

D

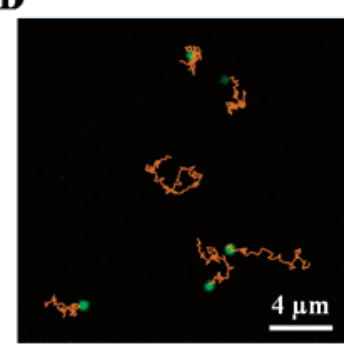

Figure 1. (A) Principle of nanoparticle-assisted surface immobilization of phospholipid liposomes. Charged nanoparticles, adsorbed to the outer surface of phospholipid vesicles, protect them against fusion with surfaces but cause attraction to surfaces of opposite charge. (B) This epifluorescence image shows nanoparticle-stabilized vesicles randomly immobilized on cationic glass at low surface coverage (see text). The liposomes were allowed to adsorb from a $1 \%$ vesicle suspension for $5 \mathrm{~min}$, and then excess liposomes were rinsed out using copious PBS buffer. The image area is $25 \mu \mathrm{m} \times 25$ $\mu \mathrm{m}$. (C) Schematic illustration of nanoparticle-stabilized liposomes meeting surfaces of the same charge. (D) In this epifluorescence image, the yellow lines indicate trajectories of individual liposomes, each step $30 \mathrm{~ms}$ long. Quantitative image analysis shows random walk behavior in this near-surface region, with the reasonable translational diffusion coefficient of $0.60 \mu \mathrm{m}^{2} /$ s. The image area is $20 \mu \mathrm{m} \times 20 \mu \mathrm{m}$.

Avanti Polar Lipids, Inc. Carboxyl-modified white polystyrene (PS) latex, whose surface was hydrophilic and negatively charged, was purchased from Interfacial Dynamics Corp. (Eugene, OR); control experiments (see below) also used cationic nanoparticles. Using methods described elsewhere, ${ }^{14}$ liposomes of DLPC, diameter 200 $\mathrm{nm}$, were prepared at 1 vol \% concentration and mixed by lowpower sonication with negatively charged PS nanoparticles, diameter $20 \mathrm{~nm}$. The molar ratio 100:1, approximately $1: 1$ by weight, corresponds to $\sim 25 \%$ surface coverage. To image the liposomes, we added a small quantity of fluorescent-labeled lipid, typically $\sim 100$ molecules per liposome on average.

For imaging, epifluorescence microscopy was employed. Light $(532 \mathrm{~nm})$ from a diode-pumped Nd:YAG laser (CrystaLaser) was directed into an inverted microscope (Zeiss Axiovert 200) in an epi-illumination geometry. Briefly, the laser was focused to the back focal point of an air objective (LD Neofluor $63 \times$, NA $=0.75$ ), causing the beam out of the objective to be parallel and homogeneous. Fluorescence emission was collected through the same objective and detected by a back-illuminated electron multiplying charge-coupled device (CCD) camera (Andor iXon DV-887 BI) after removing the laser scattering by a $532 \mathrm{~nm}$ notch filter. The total system magnification of the CCD was $150 \mathrm{~nm} \mathrm{pixel}{ }^{-1}$.

Figure 1A illustrates schematically the simplest route, surface immobilization at random spots. Liposomes of the zwitterionic lipid DLPC were stabilized with anionic nanoparticles as described above 

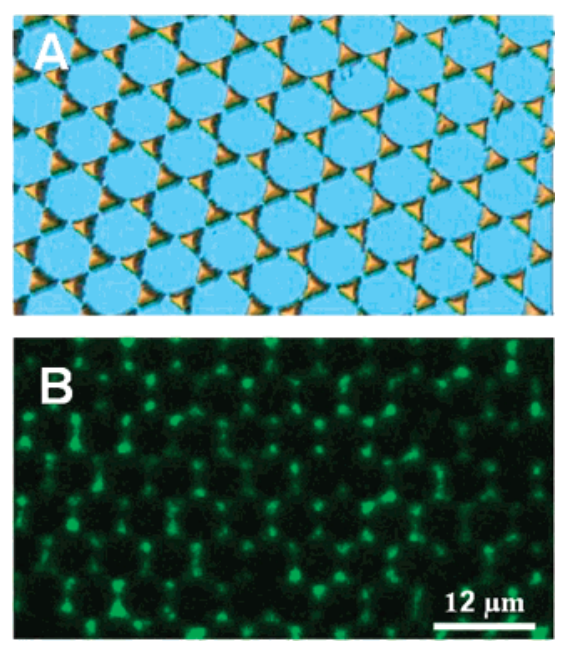

Figure 2. Nanoparticle-stabilized vesicles adsorb to form patterned surface arrays, mimicking charge arrays on the surface. (A) AFM image of a hexagonal array of cationically functionalized Au formed by microsphere lithography, leaving a negative charge in the intervening regions. (B) Nanoparticle-stabilized liposomes adsorb selectively onto these cationic patches, and none remain in the regions of negative charge. In this experiment, the patterned substrate was exposed to the vesicle suspension for $60 \mathrm{~min}$, and then the excess liposomes were rinsed out by copious PBS buffer. The image area is $70 \mu \mathrm{m} \times 45 \mu \mathrm{m}$.

and then exposed to glass uniformly coated with a positively charged monolayer (produced by prior adsorption of a cationic polyelectrolyte, quaternized poly-4-vinylpyridine); surface properties of this adsorbed polyelectrolyte were described earlier. ${ }^{16}$ The epifluorescence images in Figure 1B show the presence of immobilized vesicles; the surface coverage is small because of the short adsorption time (see figure caption). They remained stable up to the longest periods of time investigated, several days.

A second route of surface attachment has charged nanoparticles meet a surface with the same charge such that electrostatic repulsion prevents absorption (Figure 1C). Liposomes of DLPC, stabilized with anionic nanoparticles, were exposed to a surface coated with a negative charge (produced by fused quartz treated with piranha solution that was selected as substrate). Note that this is a stringent test of the efficacy of protection against fusion because quartz is commonly used as solid substrate to form supported bilayers. Figure 1D shows the trajectories observed by epifluorescence in this case. The particle-stabilized liposomes do not adsorb; they settle to the surface by gravity and then hover near it while continuing to diffuse. Image analysis using single particle tracking ${ }^{17}$ shows that the translational diffusion coefficient was $0.60 \mu \mathrm{m}^{2} \mathrm{~s}^{-1}$, a reasonable value for particles this size in bulk suspension. Further experiments found that no liposomes remained after rinsing the quartz with copious PBS buffer.

A third route of surface attachment has charged nanoparticles meet a surface patterned with alternating cationic and anionic patches such that electrostatic attraction causes localized adsorption in faithful reproduction of the charge array. We form charge arrays by microsphere lithography ${ }^{18}$ combined with gold chemical modification, producing the hexagonal pattern of gold whose AFM image is shown in Figure 2A. Basically, a monolayer of polystyrene latex spheres, $6 \mu \mathrm{m}$ in diameter, was formed on a quartz surface previously treated with piranha solution $\left(30 \mathrm{vol} \% \mathrm{H}_{2} \mathrm{O}_{2}\right.$ and 70 vol \% sulfuric acid). Electron beam evaporation (Temescal) was used to deposit first a layer of $\mathrm{Ti}(2 \mathrm{~nm})$ and then a layer of $\mathrm{Au}$
$(50 \mathrm{~nm})$. The latex spheres were removed by sonication in water, leaving gold residues in the gaps between previous placements of the microspheres. To produce areas of positive charge, the gold is functionalized with a trimethylamine thiol (11-mercapto- $\mathrm{N}, \mathrm{N}, \mathrm{N}$ trimethylundecane-1-aminium chloride, $\left.\mathrm{HS}\left(\mathrm{CH}_{2}\right)_{11} \mathrm{~N}^{+}\left(\mathrm{CH}_{3}\right)_{3} \cdot \mathrm{Cl}^{-}\right)$. Between the gold, the surface was native anionic quartz. The epifluorescence image in Figure 2B shows that nanoparticlestabilized liposomes adsorb selectively onto cationic patches and are immobilized there while the anionic patches contain no immobilized liposomes. This figure shows the large-scale liposome pattern that resulted.

These findings were general. The same holds using cationic latex nanoparticles, and also using silica nanoparticles (10 nm diameter) rather than the polystyrene latex employed in this work. In other works, we find that these methods can also be used to form largescale liposome monolayer arrays that are fully dense.

These surface-immobilized vesicles remain stable over a period of days. In the case of adsorption to a surface of opposite charge, nanoparticles on the liposome probably tend to segregate to the undersurface, leaving proteins impregnated in the upper surface even more accessible to chemical reaction from the solution side. Another promising application will be to perform chemical reactions within these nanocontainer-sized objects. Because each separate liposome is impermeable, elementary reactions, each of them performed at a dilute concentration, can be studied in parallel among many such liposomes. In addition, communication between liposomes in densely packed patterns may be possible when the vesicle is impregnated with pore proteins. Studies in this direction are in progress and will be reported presently.

Acknowledgment. We thank Stephen Anthony for particle tracking, Janet Wong for help with AFM measurements, and Huilin Tu and Paul Braun for the gift of trimethylamine thiol. This work was supported in part by the U.S. Department of Energy, Division of Materials Science, under Award No. DEFG02-02ER46019, in part through the WaterCAMPWS, a Science and Technology Center of Advanced Materials for the Purification of Water, NSF CTS0120978 .

\section{References}

(1) Pantos, A.; Tsiourvas, D.; Paleos, C. M.; Nounesis, G. Langmuir 2005 $21,6696$.

(2) Dos Santos, N.; Waterhouse, D.; Masin, D.; Tardi, P. G.; Karlsson, G.; Edwards, K.; Bally, M. B. J. Controlled Release 2005, 105, 89.

(3) Sharma, A.; Sharma, U. S. Int. J. Pharm. 1997, 154, 123.

(4) Rhoades, E.; Gussakoysky, E.; Haran, G. Proc. Natl. Acad. Sci. U.S.A. 2003, 100, 3197 .

(5) Michel, M.; Winterhalter, M.; Darbois, L.; Hemmerle, J.; Voegel, J. C.; Schaaf, P.; Ball, V. Langmuir 2004, 20, 6127.

(6) Kohl, T.; Haustein, E.; Schwille, P. Biophys. J. 2005, 89, 2770

(7) Roach, P.; Farrar, D.; Perry, C. C. J. Am. Chem. Soc. 2005, 127, 8168

(8) Jass, J.; Tjärnhage, T.; Puu, G. Biophys. J. 2000, 79, 3153

(9) Tero, R.; Takizawa, M.; Li, Y.-J.; Yamazaki, M.; Urisu, T. Langmuir 2004, 20, 7526.

(10) Yoshina-Ishii, C.; Boxer, S. G. J. Am. Chem. Soc. 2003, 125, 3696

(11) Yoshina-Ishii, C.; Miller, G. P.; Kraft, M. L.; Kool, E. T.; Boxer, S. G. J. Am. Chem. Soc. 2005, 127, 1356.

(12) Jung, L. S.; Schumaker-Parry, J. S.; Campbell, C. T.; Yee, S. S.; Gelb M. H. J. Am. Chem. Soc. 2000, 122, 4177. Boukobza, E.; Sonnenfeld, A.; Haran, G. J. Phys. Chem. B 2001, 105, 12165.

(13) Pfeiffer, I.; Höök, F. J. Am. Chem. Soc. 2004, 126, 10224.

(14) Zhang, L.; Granick, S. Nano Lett. 2006, 6, 694.

(15) Xie, A. F.; Yamada, R.; Gewirth, A. A.; Granick, S. Phys. Rev. Lett. 2002 89,246103

(16) Sukhishvili, S. A.; Granick, S. J. Chem. Phys. 1998, 109, 6861.

(17) Anthony, S.; Zhang, L.; Granick, S. Langmuir 2006, 22, 5266.

(18) Takei, H.; Shimizu, N. Langmuir 1997, 13, 1865.

JA062620R 